\title{
Digital or Analog Hough Transform?
}

\author{
N. Kiryati, M. Lindenbaum and A.M. Bruckstein *
}

\author{
Department of Electrical Engineering \\ * Department of Computer Science \\ Technion - Israel Institute of Technology \\ Haifa 32000, Israel
}

\begin{abstract}
A variation of the Hough Transform that is aimed at detecting digital lines has been recently suggested. Other Hough algorithms are intended to detect straight lines in the ana$\log$ pre-image. These approaches are analyzed and compared in terms of the relation between the achievable resolution and the required number of accumulators, using $a$ definition of resolution that is based on the Geometric Probability measure of straight lines. It is shown that the "analog" approach is greatly superior in high resolution applications, where a "digital" Hough Transform would generally require an infeasibly large number of accumulators.
\end{abstract}

The Hough Transform [2,4] is a well known technique for recognizing predefined features in edge maps. In this paper, the Hough Transform for detecting straight lines is considered.

Most Hough algorithms consist of an incrementation stage, in which each edge point "votes" for the parameter-pairs of all possible straight lines on which it can lie, and an exhaustive search for peaks. These correspond to large collinear sets of edge-points.

Originally, the slope-intercept $(m, b)$ parametrization of straight lines had been employed in the Hough Transform. It has the advantage that an edge point corresponds to a straight line in the parameter space, thus voting is simple. Its drawback is that the parameter space is unbounded, implying some theoretical and practical difficulties. With normal $(p, \theta)$ parametrization of straight lines, as suggested by [2], an edge point corresponds to a sinusoid in the parameter space, thus voting is somewhat more complex. The normal parametrization has the advantage that a bounded image leads to a bounded parameter space. Other straight-line parametrizations have also been suggested, see $[4,11,17]$.

In most implementations of the Hough algorithm the parameter space is represented by a rectangular accumulator array, such that each accumulator corresponds to a rectangular, constant size domain in the parameter space. The quantization of the parameter space greatly influences the resolution and detection capabilitics of the algorithm, as well as the computational and storage requirements; see $[6,16]$.

Errors in the location of the edge points impair the performance of conventional Hough algorithms. Such errors are usually due to the effects of image noise and distortion, including image digitization, on the output of the edge detector. Modern forms of the Hough Transform, e.g. $[6,10,15]$, provide some compensation for location crrors in the data, thus improving the performance of the algorithm. These variants do not provide special treatment for location errors which are due to image digitization; this is justified by the ability of modern edge detection schemes to offer sub-pixel accuracy when the levels of other sources of image noisc are low. In this paper, $[6,10,15]$ and related versions are referred to as "Analog Hough Transforms", since they are intended to detect straight lines in the analog "pre-image".

An interesting form of the Hough Transform has recently been described in [1]. It is specifically aimed at detecting digital straight lines [3], and is thus referred to here as the "Digital Hough Transform". This version of the Hough Transform employs the slope-intercept paramctrization, and a non-uniform parameter space quantization scheme that, in principle, assigns an accumulator to each of the triangular or quadrilateral domains in the $(m, b)$ space that correspond to distinct digital lines. Such domains have been originally described and characterized in [3]. It is claimed in [1] that the Digital Hough Transform is a computationally attractive alternative to usual high resolution implementations of the Hough Transform. The purpose of this paper is to compare the analog and digital approaches to the Hough Transform, and in particular to compare the resolution that a certain number of accumulators can "buy".

\section{DIGITAL HOUGH TRANSFORM}

A fundamental observation underlying the digital approach to the Hough Transform is that $A_{D}(N)$, the number of possible distinct digital straight lines in an $N \times N$ digital binary image, is finite. Recently, Lindenbaum, Koplowitz and Bruckstein [8] have shown that

$$
A_{D}(N)=\frac{3}{\pi^{2}} N^{4}+O\left(N^{3} \log N\right) .
$$

Dorst and Smeulders [3] have shown that each digital line corresponds to an (infinite) set of "analog" straight lines that can be represented by a distinct domain in the $(m, b)$ parameter space. A digital Hough algorithm to detect digital 
straight lines can, in principle, be constructed by assigning an accumulator to each of the $A_{D}(N)$ domains. Clever data structures [1] can reduce this number if the number of edgc pixels in the image is small.

The Digital Hough Transform is at its best when the image digitization process is the dominant source of location errors in the data points, i.e. when the image contains truc digital straight lines. It may be observed that if the task is to merely verify that a digital are is a digital straight line, then alternative, extremely efficient $O(N)$ algorithms $[7,13]$ arc available.

Unlike most conventional Hough algorithms, the "resolution" of the Digital Hough Transform given an $N \times N$ image cannot be set or modified by design, since the number of accumulators is fixed, equal to the number of possible digital straight lines in the digital image. (Decimation of the digital image would, however, allow to reduce the number of accumulators and degrade resolution.)

To enable comparison with other Hough variants, a meaningful measure of resolution must be devised. For the Digital Hough Transform a reasonable approach seems to be the quantification of the "residual ambiguity" in the position of a straight line once the digital straight line to which it relates has been identified. This requires to, somehow, measure the infinite set of straight lines that belong to the corresponding domain in the $(m, b)$ parameter space.

In the Digital Hough Transform the quantization of the $(m, b)$ parameter space is nonuniform in the sense that accumulators are assigned to domains of different sizes and shapes. One might be tempted to determine an "average" domain and perhaps regard its area as a measure of the residual ambiguity. This is, however, unacceptable since domains of equal area in different locations in the $(m, b)$ place cannot be meaningfully associated with equal residual ambiguities. Furthermore, lines in real images are usually not drawn from a uniform probability source, so there is little engineering justification in using the average as a figure of merit.

To avoid averaging, one might want to focus on the particular digital straight line that leads to the worst-case residual ambiguity. But the worst cases relate to straight lines that set very few pixels in the image, such as lines that traverse the image near its corners. The ambiguity in the location of such lines is not very interesting, so a better approach seems to be to measure the worst-case residual ambiguity among lines that intersect opposite sides of the image and set all pixels in between.

A meaningful measure of (an infinite set of) straight lines should be invariant to translation and rotation of the coordinate system. A unique measure satisfying these requirements is known in the field of Geometric Probability [12]. Two important conclusions, informally rephrased, are that patches of equal area in a $(\rho, \theta)$ normal parameter space (but not in the $(m, b)$ space!) correspond to (infinite) sets of lines of equal measure, and that the measure of straight lincs that traverse a convex region is equal to the perimeter of the region.

By the Geometric Probability measure of straight lines, the worst-case residual ambiguity in the location of lines that intersect opposite sides of the image and set all pixcls in between is clearly associated with digital lines that are parallel to one of the axcs of the grid, as shown in Figure 1. The computation of the ambiguity is described with reference to Figure 2.

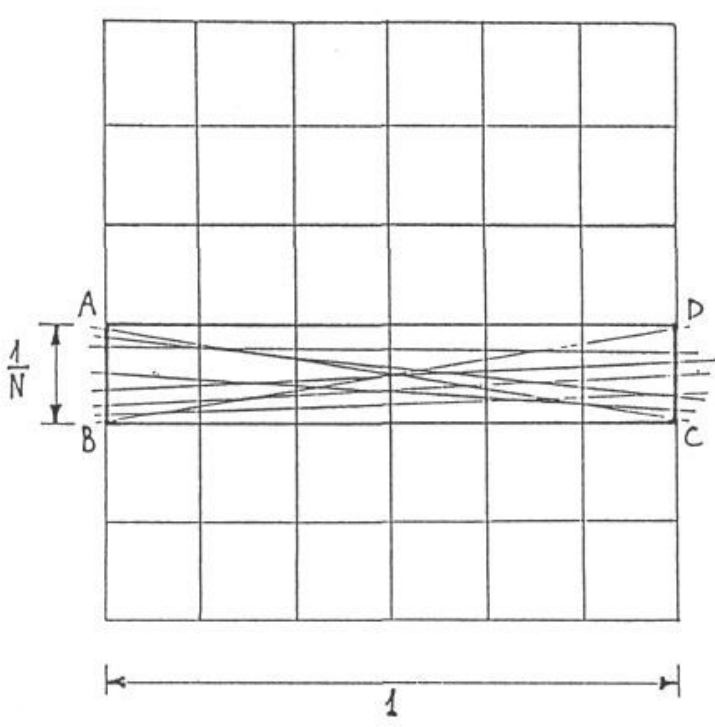

Figure 1. The digital line that induces worst-case residual ambiguity among lines that intersect opposite sides of the image and set all pixels in between.

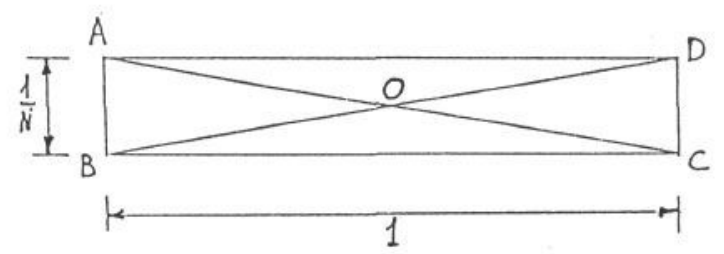

Figure 2. The geometric structures used for computing the measure of straight lines that intersect both the segment $A B$ and the segment $C D$.

Let $L_{x}$ denote the set of straight lines that intersect a gcometric feature $X$, and let $M_{x}=M\left(L_{x}\right)$ denote the measure of that set. The worst-case residual ambiguity is defined as the measure of the set of lines that intersect both segments $A B$ and $C D$ :

$$
R_{D H T}(N) \stackrel{\Delta}{=} M\left(L_{A B} \cap L_{C D}\right) .
$$

Clearly, $L_{A B} \cap L_{C D}=L_{A B O} \cap L_{C D O}$. Furthermore,

$$
M\left(L_{A B O} \cap L_{C D O}\right)=M_{A B O}+M_{C D O}-M\left(L_{A B O} \cup L_{C D O}\right)(3)
$$




$$
\begin{aligned}
\text { But } L_{A B O} \cup & L_{C D O}=L_{A B C D}, \text { so } \\
& R_{D H T}(N)=M_{A B O}+M_{C D O}-M_{A B C D} .
\end{aligned}
$$

The triangles $A B O$ and $C D O$, and the rectangle $A B C D$ are convex polygons, thus the measure of the lines that traverse each of them is simply the respective perimeter. Hence,

$$
\begin{gathered}
R_{D H T}(N)=2\left(1 / N+\sqrt{1+1 / N^{2}}\right)-(2 / N+2) \\
R_{D H T}(N)=2\left(\sqrt{1+1 / N^{2}}-1\right) .
\end{gathered}
$$

If $N$ is large the following approximation holds:

$$
R_{D H T}(N) \approx 1 / N^{2} .
$$

It is concluded that the resolution of the Digital Hough Transform is such that the worst-case residual ambiguity among lines that intersect opposite sides of the image and set all pixels in between is approximately $1 / N^{2}$ according to the rotation and translation invariant measure of straight lines. The required number of accumulators is asymptotically $3 N^{4} / \pi^{2}$.

\section{ANALOG HOUGH TRANSFORM}

By specifically tailoring the Hough algorithm to detect digital straight lines, one implicitly assumes that the discretization of the image is the dominant source for errors in the location of edge points. Very often this is not the case; the accuracy of modern edge detectors largely depends on the level of image noise, and can reach sub-pixel levels when the signal to noise ratio is high. Thus "analog" Hough algorithms, capable of detecting straight lines in the analog preimage, are useful.

In conventional Hough Transforms, errors in the coordinates of data points were altogether ignored or believed to be compensated by the quantization of the parameter space. The shortcomings of that approach manifest themselves in the spreading of peaks in the parameter space and in the appearance of false peaks. These phenomena can lead to considerable degradation in the performance of the Hough algorithm in terms of detection capability and effective resolution. Thus, the simplistic assumption that the resolution of conventional Hough algorithms is directly related to the quantization density of the parameter space fails to account for crrors in the location of edge points.

Variants of the Hough Transform that provide compensation for errors in the location of data points have been available for some time, but have usually been difficult to analyze. Recently, Kiryati and Bruckstein [6] studied an extended Hough Transform [15] that provides compensation for edge-point location errors, and using a signaltheoretic analysis were finally able to show how its resolution depends on the quantization of the parameter space, i.e. on the number of accumulators. This algorithm is thus an "analog" Hough Transform that is convenient to compare with the Digital Hough Transform with respect to the dependence of the effective resolution on the number of accumulators. Relevant ideas and results from [6] are presented and extended in the rest of this section.

Consider the Duda and Hart [2] algorithm, in which detection of collinear points is substituted by the detection of sinusoid intersections. The voting process is intended to produce at the accumulator array a discrete approximation of the continuous-support Hough Transform $h(\rho, \theta)$ defined as follows: Let $p_{i} \geq 0$ and $0 \leq \theta_{i}<2 \pi$ denote the polar coordinates of any edge point $p_{i} \in P$. Then every $p_{i} \in P$ generates a sinusoid $\rho_{i}^{0}(\theta)$ in the $(\rho, \theta)$ parameter plane:

$$
\rho_{i}^{0}(\theta)=\rho_{i} \cos \left(\theta_{i}-\theta\right) \quad \theta \in[0, \pi) .
$$

An indicator function is associated with each sinusoid:

$$
I_{i}^{0}(\rho, \theta)= \begin{cases}1 & \rho=\rho_{i}^{0}(\theta) \\ 0 & \text { otherwise }\end{cases}
$$

Summing up the indicator functions yields the continuoussupport Hough Transform:

$$
h(\rho, \theta)=\sum_{i} I_{i}^{0}(\rho, \theta) .
$$

$h(\rho, \theta)$ is a discontinuous - hence non-bandlimited - function. This is not changed by the spatial-dependent transformation inherent in the voting process. It is well known that due to aliasing effects a non-bandlimited signal cannot be properly represented by a discrete set of samples.

This basic inadequacy in the algorithm was studied in depth [6]; the key to the solution is the replacement of $h(\rho, \theta)$ by an "almost" bandlimited function, such that "sufficient" parameter-space sampling can be carried out. Interpolation by an appropriate low-pass filter then allows high resolution search for maxima in the parameter space. The smoothing of $h(p, \theta)$ must be performed before sampling, in a way that preserves the useful properties of the algorithm in line detection, and in a computationally feasible manner.

In particular, it has been suggested $[6,15]$ that the indicator function (9) be replaced by

$$
I_{i}(\rho, \theta)=C\left(\rho-\rho_{i}^{0}(\theta)\right)
$$

where $C(\cdot)$ is an "influence function" to be discussed in the sequel. This results with a modified continuous-support Hough Transform

$$
z(\rho, \theta)=\sum_{i} I_{i}(\rho, \theta) .
$$

$z(\rho, \theta)$ can be point-sampled by assigning accumulators to a discretc set of sampling points, and cvaluating (12) only at these points.

The replacement of (9) and (10) by (11) and (12) can be 
visualized as the replacement of the sinusoids in the Duda and Hart algorithm by sinusoidal bands whose vertical profile is $C(\cdot)$. If $C(\cdot)$ has finite support, the algorithm remains computationally feasible (and apt for parallelization).

The introduction of the influence function $C(\cdot)$ into the Duda and Hart algorithm is equivalent to surrounding cach edge-point by a circularly symmetric density distribution (which is the inverse Abel Transform of the influence function) and replacing the Duda and Hart Transform by the Radon Transform. By choosing $C(\cdot)$ to be symmetric, positive, reasonably "well behaved" and of finite support $\left(-r_{m}, r_{m}\right)$, the useful properties of the algorithm are maintained, and compensation for location errors (limited to $r_{m}$ ) is provided.

Kiryati and Bruckstein [6] have shown that if $C(\cdot)$ is bandlimited to $\left(-\omega_{m}, \omega_{m}\right)$, then the modified continuoussupport Hough Transform $z(\rho, \theta)$ is also 2-D bandlimited, and can be fully represented by a discrete set of samples. The uncertainty principle of signal representation dictates, however, that the influence function $C(\cdot)$ and its Fourier Transform cannot both be of "short duration". Since implementation of the modified Hough Transform implies an influence function $C(\cdot)$ of finite support, the use of support limited influence functions that have the smallest possible "effective bandwidth" in a certain sense has been suggested, e.g.

$$
C(r)= \begin{cases}k \cos \left(\pi r / 2 r_{m}\right) & |r| \leq r_{m} \\ 0 & |r|>r_{m}\end{cases}
$$

where $k$ is a constant. With $k>0$ this influence function is a positive, symmetric and monotonically decreasing function of $|r|$. Furthermore, it has the smallest possible effective bandwidth (in the sense of the second order energy moment of its Fourier Transform) among all functions that are support limited to $r_{m}$.

With a support limited influence function which is also effectively bandlimited, $z(\rho, \theta)$ is effectively bandlimited, has finite support in the $\rho$ direction and is periodic in the $\theta$ direction. Thus it can be represented by a finite set of point samples with negligible aliasing. It has been shown that due to the bow-tie shaped band-region of $z(\rho, \theta)$, optimal sampling of $z(\rho, \theta)$ is on an hexagonal grid, and sampling on a rectangular grid doubles the sampling requirement, i.e. the required number of accumulators.

Assuming a circular image of radius $\rho_{m}$, Kiryati and Bruckstein [6] have shown that the minimum required number of accumulators is

$$
\left.A_{A}^{o}=\left(\rho_{m}+r_{m}\right) \omega_{m}\left(\left\lfloor\rho_{m} \omega_{m}\right\rfloor\right)+3\right) i \pi
$$

where $\lfloor x\rfloor$ is the largest integer equal to or smaller than $x$. To compare with the Digital Hough Transform a unit square image is considercd. A unit square circumscribes a circle of radius 0.5 and is inscribed in a circle of radius $\sqrt{2} / 2$. Reasonably assuming $r_{m} \ll 1$, it immediately follows that $A_{A}$, the minimum requircd number of samples in this "analogtype" Hough Transform satisfies

$$
A_{A}=a \frac{\omega_{m}^{2}}{2 \pi}
$$

where $0.5<a<1$ is a constant. Using the influence function (13) and the convention that its effective bandwidth is triple the square root of the normalized second order energy moment of its Fourier Transform,

$$
\omega_{m}=3 \pi / 2 r_{m} .
$$

Therefore, the minimum required number of accumulators is:

$$
A_{A}=a \frac{9 \pi}{8 r_{m}^{2}}
$$

where $r_{m}$ is the radius of support of the influence function $C(\cdot)$, and represents the ambiguity in the location of the data points. Hence, the resolution of this version of the Hough Transform is upper-bounded either by the assumed magnitude of edge-point location errors (forcing certain $r_{m}$ and $A_{A}$ ) or by the available number of accumulators $A_{A}$, forcing $r_{m}$ to be larger than a certain minimum.

To compare with the Digital Hough Transform, we proceed to determine (in terms of $r_{m}$, and through (17) in terms of $A_{A}$ ) the worst-case ambiguity in lines that intersect opposite sides of the image and set all pixels in between. As in the case of the Digital Hough Transform, the worst-case corresponds to lines that are parallel to one of the image sides and yield collinear edge-points. See Figure 3.

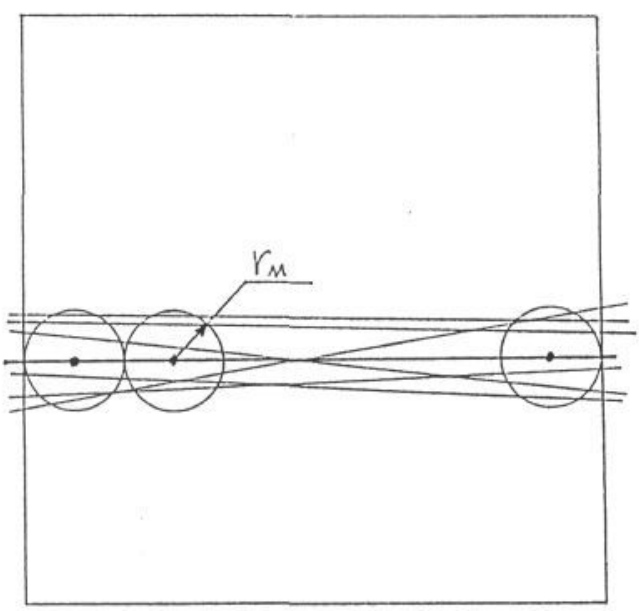

Figure 3. In the Analog Hough Transform [6], the worstcase ambiguity among lines that intersect opposite sides of the image and set all pixels in between is associated with lines that are parallel to one of the coordinate axes.

If $r_{m} \ll 1$ as assumed, one can simply substitute

$$
1 / N=2 r_{m}
$$

in (7), to obtain 


$$
R_{A H T} \approx 4 r_{m}^{2} \text {. }
$$

This means that the resolution of this Analog Hough Transform is such that the worst-case ambiguity among lines that intersect opposite sides of the image is approximately $4 r_{m}^{2}$ according to the rotation and translation invariant measure of straight lines. The minimum required number of accumulators is $9 a \pi / 8 r_{m}^{2}$, where $0.5<a<1$ is a constant. (Note that if the errors in the location of the data points are mainly due to digitization, choose $r_{m} \approx 1 / 2 N$ ).

\section{CONCLUSIONS}

In this paper digital and analog Hough algorithms are compared. The Digital Hough Transform is aimed at detecting digital straight lines; it implicitly assumes that the discretization of the image is the dominant source for cdge-point location errors. When the image is corrupted with noise, or when edge detectors capable of sub-pixel accuracy are employed, that assumption is not valid. The term "Analog Hough Transform" refers to algorithms that are aimed at detecting straight lines in the analog "pre-image". In particular, the digital approach is advocated by [1]; analog Hough algorithms are represented by the algorithm of [6].

The comparison is made in terms of the relation between the achievable resolution in each of the approaches as a function of the number of accumulators. In the Digital Hough Transform, the resolution and the required number of accumulators are fixed and governed by the dimensions of the digital image, which can only be coarsely modified by decimation. In Analog Hough Transforms the number of accumulators (and the resulting resolution) can be set by design according to specifications.

Resolution is defined in terms of the worst-case ambiguity in the location of straight lines that intersect opposite sides of the image and set all pixels in between. Ambiguity is measured by the translation and rotation invariant measure of straight lines [12]. A similar approach was used in [5] to quantitatively evaluate digitization schemes. Worst-case analysis is convenient and usually meaningful in terms of engineering specifications.

In the Digital Hough Transform of an $N \times N$ image the ambiguity $R_{D H T}(N)$ is given by (7), and $A_{D}(N)$, the required number of accumulators, is given by (1). Asymptotically,

$$
A_{D}(N) \propto 1 / R_{D H T}^{2}(N)
$$

In the Analog Hough Transform (of [6]) the ambiguity $R_{A H T}$ is given by (19), and $A_{A}$, the minimum required number of accumulators is given by (17). It follows that

$$
A_{A} \propto 1 / R_{A H T} .
$$

The comparison between (20) and (21) reveals that, in high resolution applications, the Analog Hough Transform is markedly superior to the Digital Hough Transform in terms of the rcsolution that an added accumulator "buys".

In the Digital Hough Transform the tessalation of the parameter space is very non-uniform, meaning that certain digital lines correspond to small sets of lines in the preimage, while other digital lines, that constitute the worstcase, correspond to large sets of lines in the pre-image. The "Muff" Transform [17] is a variant of the Hough Transform that has some "digital flavour", yet achieves better uniformity in the tessalation of the parameter space. An important contribution of [1] is in providing a conceptual link between the theory of Digital Geometry and Hough Transform rescarch.

\section{Acknowledgement}

The authors thank Dr. V.F. Leavers, King's College, London, for bringing reference [1] to their attention.

\section{REFERENCES}

1. Cyganski D. Noel W.F. and Orr J.A. "The Analytic Hough Transform", Proc. SPIE/SPSE Symposium on Electronic Imaging, Santa Clara (1990).

2. Duda R.O. and Hart P.E. "Use of the Hough Transform to Detect Lines and Curves in Pictures", Commun. ACM, Vol. 15 (1972) pp 11-15.

3. Dorst L. and Smeulders A.W.M. "Discrete Representation of Straight Lines", IEEE Trans. on Pattern Analysis and Machine Intelligence, Vol. PAMI-6 (1984) pp 450-463.

4. Illingworth J. and Kittler J. "A Survey of the Hough Transform", Computer Vision Graphics and Image Processing, Vol. 44 (1988) pp 87-116.

5. Kiryati N. and Bruckstein A.M. "Gray Levels Can Improve the Performance of Binary Image Digitizers", Proc. IEEE Computer Society Conf. on Computer Vision and Pattern Recognition, Ann Arbor (1988). Accepted to CVGIP: Graphical Models and Image Processing.

6. Kiryati N. and Bruckstein A.M. "Antialiasing the Hough Transform", Proc. 6th Scandinavian Conf. on Image Analysis, Oulu, Finland (1989). Accepted to CVGIP: Graphical Models and Image Processing.

7. Lindenbaum M. and Bruckstein A.M. "On Recursive $O(N)$ Partitioning of a Digitized Curve into Digital Straight Segments", Technical Report No. 750, Department of Electrical Engineering, Technion, Haifa 32000, Israel (1990). 
8. Lindenbaum M. Koplowitz J. and Bruckstein A.M. "On the Number of Digital Straight Lines on an $N \times N$ Grid", Proc. IEEE Computer Society Conf. on Computer Vision and Pattern Recognition, Ann Arbor (1988).

9. Melter R.A. and Rosenfeld A. "New Views of Linearity and Connectedness in Digital Geometry", Pattern Recognition Letters, Vol. 10 (1989) pp 9-16.

10. Niblack W. and Petkovic D. "On Improving the Accuracy of the Hough Transform: Theory, Simulations, and Experiments", Proc. IEEE Computer Society Conf. on Computer Vision and Pattern Recognition, Ann Arbor (1988).

11. Risse T. "Hough Transform for Line Recognition: Complexity of Evidence Accumulation and Cluster Detection", Computer Vision Graphs and Image Processing, Vol. 46 (1989) pp 327-345.

12. Santalo L. Integral Geometry and Geometric Probability, Encyclopedia of Mathematics and its Applications, Vol 1, Addison-Wesley, Reading, MA (1976).

13. Smeulders A.W.M. and Dorst L. "Decomposition of Discrete Curves into Piece-wise Straight Segments in Linear Time". Submitted for publication.

14. Svalbe I.D. "Natural Representations for Straight Lines and the Hough Transform on Discrete Arrays", IEEE Trans. on Pattern Analysis and Machine Intelligence, Vol. PAMI-11 (1989) pp 941-950.

15. Thrift P.R. and Dunn S.M. "Approximating PointSet Images by Line Segments Using a Variation of the Hough Transform", Computer Vision Graphics and Image Processing, Vol. 21 (1983) pp 383-394.

16. Van-Veen T.M. and Groen F.C.A. "Discretization Errors in the Hough Transform", Pattern Recognition, Vol. 14 (1981) pp 137-145.

17. Wallace R.S. "A Modified Hough Transform for Lines", Proc. IEEE Computer Society Conf. on Computer Vision and Pattern Recognition, pp 665-667 (1985). 